\title{
The effects of acute hypoglycaemia on memory acquisition and recall and prospective memory in type 1 diabetes
}

\author{
R. E. Warren • N. N. Zammitt • I. J. Deary • B. M. Frier
}

Received: 23 May 2006 / Accepted: 10 October 2006 / Published online: 2 December 2006

(C) Springer-Verlag 2006

\begin{abstract}
Aims/hypothesis Global memory performance is impaired during acute hypoglycaemia. This study assessed whether moderate hypoglycaemia disrupts learning and recall in isolation, and utilised a novel test of prospective memory which may better reflect the role of memory in daily life than conventional tests.

Subjects and methods Thirty-six subjects with type 1 diabetes participated, 20 with normal hypoglycaemia awareness (NHA) and 16 with impaired hypoglycaemia awareness (IHA). Each underwent a hypoglycaemic clamp with target blood glucose $2.5 \mathrm{mmol} / \mathrm{l}$. Prior to hypoglycaemia, subjects attempted to memorise instructions for a prospective memory task, and recall was assessed during hypoglycaemia. Subjects then completed the learning and immediate recall stages of three conventional memory tasks (word recall, story recall, visual recall) during hypoglycaemia. Euglycaemia was restored and delayed memory for the conventional tasks was tested. The same procedures were completed in euglycaemic control studies (blood glucose $4.5 \mathrm{mmol} / \mathrm{l})$.

Results Hypoglycaemia impaired performance significantly on the prospective memory task $(p=0.004)$. Hypoglycaemia also significantly impaired both immediate and delayed recall for the word and story recall tasks $(p<0.01$ in each case). There was no significant deterioration of performance on the visual memory task. The effect of hypogly-
\end{abstract}

R. E. Warren $\cdot$ N. N. Zammitt $\cdot$ B. M. Frier $(\bowtie)$

Department of Diabetes, Royal Infirmary of Edinburgh,

51 Little France Crescent,

Edinburgh EH16 4SA, UK

e-mail: brian.frier@luht.scot.nhs.uk

I. J. Deary

Department of Psychology, University of Edinburgh,

Edinburgh, UK caemia did not differ significantly between subjects with NHA and IHA.

Conclusions/interpretation Impaired performance on the prospective memory task during hypoglycaemia demonstrates that recall is disrupted by hypoglycaemia. Impaired performance on the conventional memory tasks demonstrates that learning is also disrupted by hypoglycaemia. Results of the prospective memory task support the relevance of these findings to the everyday lives of people with diabetes.

Keywords Cognitive function · Hypoglycaemia . Hypoglycaemia awareness · Memory · Type 1 diabetes
Abbreviations
AVLT Auditory Verbal Learning Test
IHA impaired hypoglycaemia awareness
NHA normal hypoglycaemia awareness

\section{Introduction}

Hypoglycaemia is a common side effect of insulin therapy for type 1 diabetes [1]. Experimentally induced acute hypoglycaemia causes a deterioration in performance on a wide range of cognitive tasks in humans [2]. Memory is one the of the most important cognitive domains with respect to everyday function. Previous studies applied memory tests as part of a larger battery of cognitive tests during hypoglycaemia [3-8], and the variability of reported results may relate to the use of different memory tests. Methodological variation in hypoglycaemia studies has been reviewed elsewhere [9]; particularly pertinent issues include sample size and statistical power, the method of 
measurement of blood glucose, and the target level for, and the duration of, hypoglycaemia.

Neuropsychological research indicates that memory comprises a number of subsystems, including sensory memory, short-term memory and long-term memory [10]. We have studied previously the effects of hypoglycaemia on various components of memory using consistent experimental techniques and validated memory tasks. Hypoglycaemia impairs the auditory and visual processing subsystems of sensory memory, short-term memory, working memory and delayed memory in both diabetic and nondiabetic subjects [11-16]. Working memory and delayed memory are particularly susceptible to hypoglycaemia [17].

In all previous studies, both the acquisition (learning) and recall of material took place during hypoglycaemia. Deterioration in memory performance during hypoglycaemia may have resulted from impairment of learning, consolidation (transfer of information from short- to longterm memory), recall, or any combination of these. The present study was designed to investigate whether these processes are independently sensitive to hypoglycaemia by separating the acquisition and recall phases of memory tasks so that only one occurred during hypoglycaemia.

Conventional laboratory memory tests tend to involve tasks such as learning lists of words, prose passages, and geometrical shapes. Such tests may not reflect the memory demands of daily living, which require coordination of multiple cognitive processes such as planning and vigilance, and therefore may not have ecological relevance $[18,19]$. Recall of retrospective events has been distinguished from prospective memory (remembering intended actions, or 'remembering to remember'), and tests of prospective memory may have greater ecological validity [20]. Titov and Knight developed a novel test of prospective memory in which subjects memorised a shopping list and then viewed a video of a pedestrian journey around an urban shopping area, reporting items to be purchased when specified shops appeared [21]. This videobased memory task had good test-retest reliability. It also had good criterion validity: it correlated well with performance on the same task when subjects actually walked through the shopping area. Comparative scores were obtained for 35 subjects, yielding a correlation of $r=0.71, p<0.001$, and no significant effect of study type (video vs in vivo) in ANOVA. A similar video task was created for the present study to explore the effects of hypoglycaemia on prospective memory in a practical setting.

Evidence has accumulated to show that recurrent exposure to hypoglycaemia in people with type 1 diabetes results in cerebral adaptation, such that cognitive performance is relatively preserved during hypoglycaemia [2227]. Repeated exposure to hypoglycaemia can also result in impaired hypoglycaemia awareness, and a shared mechanism for the two phenomena has been proposed [28]. Thus, in studies of people with type 1 diabetes, it is important that subjects with impaired hypoglycaemia awareness (which may be a surrogate marker of cerebral adaptation to hypoglycaemia) are considered separately from those with normal hypoglycaemia awareness.

The aims of the present study were: (1) to assess the effects of acute hypoglycaemia on different memory processes (acquisition and recall) for conventional memory tasks; (2) to assess the effects of acute hypoglycaemia on a novel prospective memory task; and (3) to compare these effects in people with type 1 diabetes who had either normal or impaired hypoglycaemia awareness.

\section{Subjects and methods}

The study protocol was approved by the Lothian Medical Research Ethics Committee, and all subjects gave informed consent to participation.

\section{Subjects}

Subjects were required to have a diagnosis of type 1 diabetes, age between 18 and 45 years, a BMI between 20 and $30 \mathrm{~kg} / \mathrm{m}^{2}$, and $\mathrm{HbA}_{1 \mathrm{c}}$ values between 7 and $10 \%$. They were ineligible if they had any significant current medical condition or contraindication to experimental hypoglycaemia. Female patients were eligible only if a pregnancy test was negative.

Potential subjects were asked to grade their hypoglycaemia awareness on a scale from 1 to 7 [29], and their hypoglycaemia history was also discussed. People who chose a grade of 1 or 2 and reported no history of severe hypoglycaemia or significant change in their warning symptoms were categorised as having normal hypoglycaemia awareness (NHA). People who chose a grade between 3 and 7 and reported diminution of hypoglycaemic symptoms and episodes of unrecognised hypoglycaemia were categorised as having impaired hypoglycaemia awareness (IHA) [29]. People whose self-rated awareness appeared inconsistent with their hypoglycaemia history were ineligible, on the grounds that their awareness status was uncertain.

In total, 36 subjects were recruited (20 NHA, 16 IHA); their characteristics are given in Table 1. Microvascular complications were defined as any clinical diagnosis of diabetic retinopathy, neuropathy or nephropathy, the latter also requiring a urine albumin:creatinine ratio persistently above the local reference maximum or serum creatinine $>150 \mu \mathrm{mol} / \mathrm{l}$. The IHA group had significantly longer duration of diabetes $(t=3.937, d f=34, p<0.001)$ and more microvascular complications $\left(\chi^{2}=5.994, d f=1, p=0.013\right)$. Other comparisons were non-significant. 
Table 1 Subject characteristics

\begin{tabular}{lll}
\hline & \multicolumn{2}{l}{ Hypoglycaemia awareness } \\
\cline { 2 - 3 } & Normal & Impaired \\
\hline$n$ & 20 & 16 \\
Male, female $(n)$ & 12,8 & 6,10 \\
Age (years): median (range) & $29(19-44)$ & $33.5(22-43)$ \\
Diabetes duration (years): median (range) & $3.8(1.1-20)$ & $15.5(2-35)$ \\
$\mathrm{HbA}_{1 \mathrm{c}}(\%)^{\mathrm{a}}$ & $7.8 \pm 1.3$ & $8.4 \pm 1.8$ \\
$\mathrm{BMI}^{2}\left(\mathrm{~kg} / \mathrm{m}^{2}\right)$ & $25.8 \pm 2.2$ & $26.8 \pm 3.6$ \\
Subjects with microvascular & $1(5)$ & $6(38)$ \\
complications (\%) & & \\
\hline
\end{tabular}

Data are mean \pm SD unless stated otherwise

${ }^{\text {a }}$ Non-diabetic reference range is $5.0-6.5 \%$

Glucose clamp procedure

Each subject underwent one hypoglycaemic and one euglycaemic glucose clamp, separated by at least 2 weeks. Subjects were not informed of the order in which these occurred. On the evenings before studies, subjects took their normal insulin, fasted from $22.00 \mathrm{~h}$ (consuming only water) and omitted insulin on the morning of the study. Subjects were encouraged to avoid hypoglycaemia during the $48 \mathrm{~h}$ before the planned studies by reducing insulin doses if necessary. Studies were postponed if subjects detected any episode of hypoglycaemia by symptoms or routine blood testing during this period; this resulted in six postponements (two NHA, four IHA).

An antecubital vein and a dorsal hand vein were cannulated in the non-dominant arm for infusions and blood sampling. The hand was placed within a heated blanket to arterialise venous blood. Arterialised venous samples were drawn every $5 \mathrm{~min}$ for measurement of whole blood glucose (YSI 2300 STAT analyser, Yellow Springs, $\mathrm{OH}$, USA). An infusion of soluble human insulin (Actrapid; NovoNordisk, Crawley, UK) was started at $1.5 \mathrm{mU} \mathrm{kg}^{-1}$ $\min ^{-1}$, and $20 \%$ glucose solution was infused at a variable rate to achieve the desired blood glucose concentrations.

The blood glucose concentration was initially stabilised at $4.5 \mathrm{mmol} / 1$ (euglycaemia), and maintained for $40 \mathrm{~min}$. During the hypoglycaemia studies, the blood glucose was then lowered over $20 \mathrm{~min}$ to $2.5 \mathrm{mmol} / \mathrm{l}$ and maintained for $60 \mathrm{~min}$, then raised to $4.5 \mathrm{mmol} / \mathrm{l}$ and maintained for a further $75 \mathrm{~min}$. Blood glucose was maintained at $4.5 \mathrm{mmol} / \mathrm{l}$ throughout the euglycaemia studies.

\section{Memory tests}

Two parallel versions of each test were available, which were combined to give two batteries. The order in which subjects were exposed to these batteries and the hypoglycaemia-

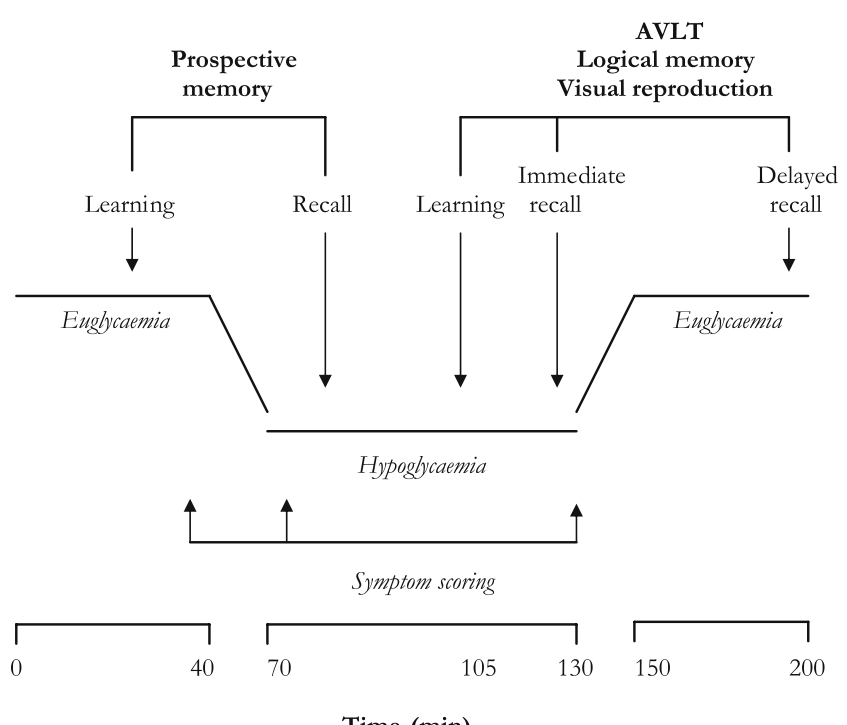

Time (min)

Fig. 1 Study outline. The same timing was adhered to for euglycaemia studies

euglycaemia order were counterbalanced within the NHA and IHA groups. The study outline is given in Fig. 1.

Prospective memory This was a novel test, based on a method developed by Titov and Knight [21]. Two videos showing the view of a pedestrian journeying around central Edinburgh were created, and for each a 'shopping list' of 21 tasks was written on 21 cards. These were made up of 'buy' tasks (e.g. 'Buy tennis balls at A\&B Sports'), 'do' tasks (e.g. 'Book a table at Smith's restaurant') and 'question' tasks (e.g. 'What is the advertised loan rate at the Mercantile Bank?'). Subjects were asked to read the cards twice at a normal reading pace, placing each card face down once it had been read. Subjects then watched the video and scored points for answering questions or stating actions at the relevant places. These were not brought to subjects' attention, and points were not awarded for answers given at inappropriate times. Pilot studies indicated that the two videos were of equivalent difficulty. Each lasted approximately $10 \mathrm{~min}$, and were similar in terms of the number of shops passed (about 80). In the present study, subjects read the cards (learning) during initial euglycaemia, and recall was tested after approximately $40 \mathrm{~min}$ during the experimental period of induced hypoglycaemia/ euglycaemia. Thus, the experimental manipulation examined the effects of hypoglycaemia on recall.

Conventional memory tests For the following memory tests, acquisition and immediate recall were tested during the experimental hour (euglycaemia or hypoglycaemia). Delayed recall was tested 90 min later during euglycaemia, without further exposure to the learned material. Thus, the 
experimental manipulation here examined the effects of hypoglycaemia on acquisition (learning).

(1) Auditory Verbal Learning Test (AVLT)-immediate and delayed [30]. Fifteen words were read to the subject, who tried to recall the words immediately. This was repeated a further four times, and the immediate recall score was the sum of correct responses for the five trials. The delayed recall score was the number correctly recalled from a single trial.

(2) Logical Memory Test-immediate and delayed. In this subtest from the Wechsler Memory ScalesRevised [31], a short story was read to the subject, who tried to recount it immediately. Points were obtained for recollection of specific details and story themes.

(3) Visual Reproduction-immediate and delayed, from the Wechsler Memory Scales-Revised [31]. Each of five line drawings was shown to the subject for $10 \mathrm{~s}$ and then hidden, and the subject tried to reproduce the drawing from memory. Reproductions were scored according to strict criteria.

\section{Symptom scores}

Subjects scored their symptoms of hypoglycaemia at baseline and during the experimental period using the Edinburgh Hypoglycaemia Scale [32].

Statistical analysis

Scores on memory tasks were compared using repeatedmeasures ANOVA. Memory test score during euglycaemia versus hypoglycaemia was the repeated measure (withinsubjects factor). Hypoglycaemia awareness, order of hypoglycaemia-euglycaemia and order of test battery were between-subjects factors. Statistical significance was accepted at $p<0.05$ and $\eta^{2}$ was used to indicate effect size. Analyses were performed using SPSS 11.0 (SPSS Inc., Chicago, IL, USA).

\section{Results}

Blood glucose

During the hypoglycaemic condition, mean \pm SD blood glucose was $2.5 \pm 0.2 \mathrm{mmol} / 1$ in the NHA group, and $2.5 \pm 0.2 \mathrm{mmol} / 1$ in the IHA group $(p=0.468)$. During euglycaemia, mean blood glucose concentration was $4.5 \pm$ $0.2 \mathrm{mmol} / 1$ (NHA) and $4.5 \pm 0.3 \mathrm{mmol} / 1(p=0.643)(\mathrm{IHA})$.

\section{Symptoms}

Total symptom scores did not change during euglycaemia. During the hypoglycaemic condition, mean symptom scores rose in both the NHA group (baseline $23.2 \pm 4.4$ vs experimental $44.1 \pm 22.2 ; p<0.001)$ and the IHA group $(22.9 \pm 7.0$ vs $28.8 \pm 8.3 ; p=0.001)$. The increment in symptom scores was significantly greater in the NHA group (interaction between glycaemic condition and awareness status, $p=0.002$ ).

Memory tasks

Results are given in Table 2. There were no significant effects of order of exposure to glycaemic condition or test battery.

Comparison of the effect of hypoglycaemia in NHA and IHA subjects The interaction between glycaemic state and hypoglycaemia awareness designation (hereafter referred to as the glycaemia $\times$ awareness interaction) was not significant for any tests. This means that there was no significant difference in the effects of hypoglycaemia on cognitive function between the NHA and IHA groups. In some cases, there was a statistically significant effect of hypoglycaemia in one group but not the other; however, in each case hypoglycaemia was associated with poorer performance in both groups, and achievement of statistical significance in one group but not the other may have been due to chance. In the absence of significant glycaemia $\times$ awareness interactions, the effect of hypoglycaemia on memory performance was determined for all subjects combined.

Prospective memory Recall was significantly impaired in all patients combined during hypoglycaemia $\left(\eta^{2}=0.255\right.$, $p=0.004)$. IHA subjects performed better overall than NHA subjects $(p=0.018)$.

Immediate verbal memory Immediate recall was significantly impaired during hypoglycaemia for the AVLT $\left(\eta^{2}=0.268, p=0.003\right)$ and logical memory test $\left(\eta^{2}=0.233\right.$, $p=0.007)$ in all subjects combined. The IHA group performed significantly better than the NHA group on the logical memory task $(p=0.030)$.

Delayed verbal memory Delayed recall was significantly impaired during hypoglycaemia for both AVLT $\left(\eta^{2}=0.373\right.$, $p<0.001)$ and logical memory test $\left(\eta^{2}=0.315, p<0.001\right)$ in all subjects combined. Again, the IHA group performed better overall on the logical memory task $(p=0.031)$. Hypoglycaemia had no effect on the proportion of information retained (delayed score as a percentage of immediate score). 


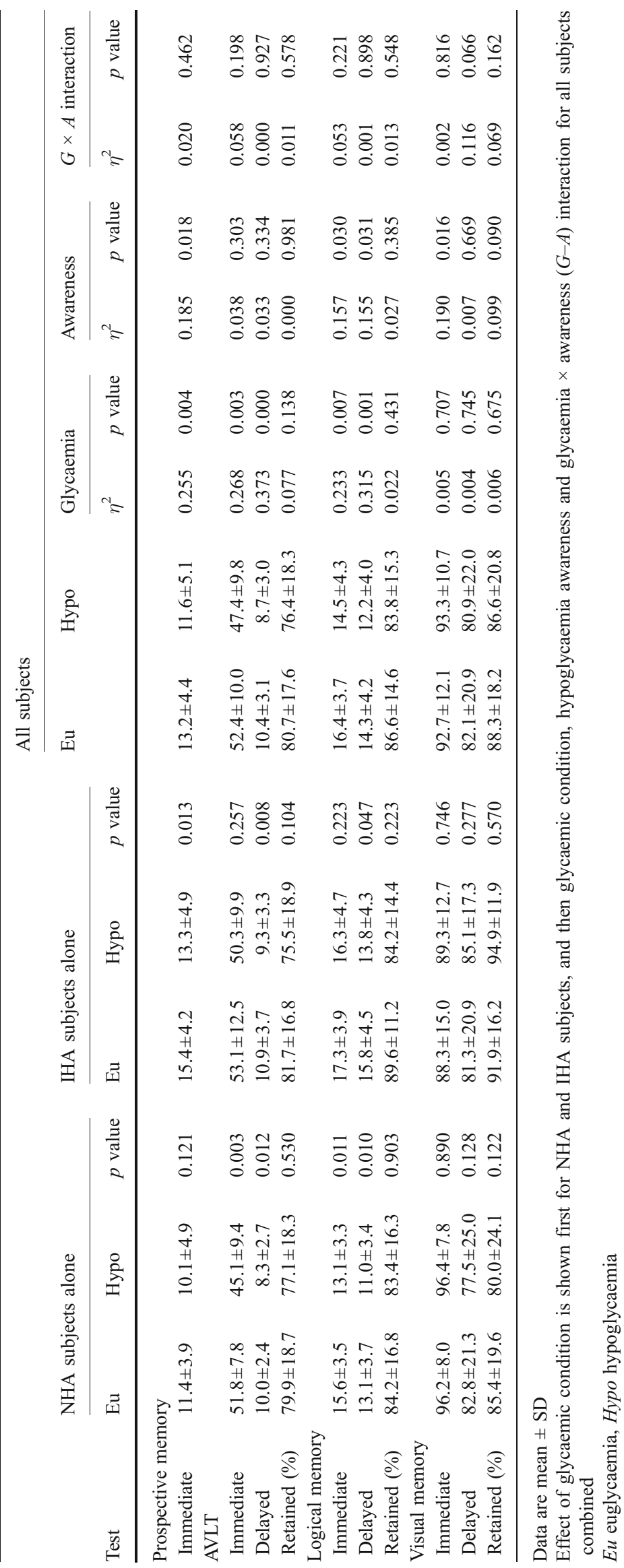


Visual memory No significant effects of hypoglycaemia were seen. Mean euglycaemia scores of 96.2 (NHA) and 88.3 (IHA) from a maximum of 104 indicated marked ceiling effects. The NHA group scored better $(p=0.016)$ on immediate visual memory.

\section{Discussion}

Performance on the novel test of prospective memory was impaired significantly during the hypoglycaemia studies and, as learning always took place during euglycaemia, the results indicate that recall had been disrupted by hypoglycaemia. The effect size of hypoglycaemia on prospective memory scores $\left(\eta^{2}\right)$ was similar to that for the conventional memory tests, which suggests that it is a similarly sensitive measure of hypoglycaemic memory dysfunction. The prospective memory task is also intuitively more reflective of memory in daily life, where list-learning is rarely relevant. In the paper upon which it was based, performance on the video task correlated well with performance on the same task in a real-life setting [21]. The data suggest that people with diabetes may suffer inability to act on previously made plans, and thus effectively to organise aspects of their daily life, as a consequence of hypoglycaemia.

Some limitations of the prospective memory task must be considered [21]. First, subjects are unable to explore the environment to obtain further cues if they suspect that an action is to be performed at a location, but cannot fully remember it. Second, they are unable to retrace their steps if an action is recalled after the location has been passed. Third, to make the task sufficiently difficult, more items are given than subjects would attempt to remember in real life. Fourth, subjects are unable to prioritise items and plan their journey. These points reflect the fact that in real life, prospective memory performance is not simply determined by accuracy of response to cues, but is also dependent on organisational and other strategies. Thus, the effect of hypoglycaemia on real-life prospective memory may be more or less than suggested by the present study, depending on whether these strategies are themselves sensitive or resistant to hypoglycaemia.

It is also possible that poorer performance during hypoglycaemia was caused by impairment of cognitive functions other than memory. The video required sustained attention for around $10 \mathrm{~min}$, and attention has been shown to be impaired during hypoglycaemia [33, 34]. Deterioration of visual information processing may have reduced the subjects' ability to glean information from the video [11, 12]. Alternatively, the results may have resulted from a general effect on cognition, as very few cognitive abilities that have been tested during hypoglycaemia have not shown deterioration [2]. These distinctions may be neuropsychological rather than practical; whatever the mechanism, hypoglycaemia impaired the ability to act on information learned previously, which in everyday life would be perceived to be a memory problem.

Immediate recall performance for the two verbal memory tasks (AVLT and logical memory) was impaired significantly during hypoglycaemia, which is consistent with previous studies [15, 16]. Delayed recall was always tested during euglycaemia, and it can be inferred that the significant impairment in delayed recall following hypoglycaemia resulted from a hypoglycaemic effect on learning and/or consolidation. A 'hangover' effect of hypoglycaemia is unlikely to be responsible, as delayed recall was tested $50 \mathrm{~min}$ after restoration of euglycaemia. The only robust study to show residual cognitive impairment demonstrated this $20 \mathrm{~min}$ after hypoglycaemia [35]; earlier studies reported impaired cognitive function 45-90 min after hypoglycaemia [8, 36-40], but each had a methodological deficiency, such as failure to test statistical significance or the absence of a euglycaemia control arm.

Correction of hypoglycaemia took approximately $20 \mathrm{~min}$, the limiting factors being the continuing insulin infusion and the maximum infusion rate of hypertonic glucose solution. The possibility that hypoglycaemia impaired performance through an effect on consolidation in the immediate postlearning period cannot be excluded. However, on this basis it is also relatively unimportant to separate learning and immediate consolidation, as hypoglycaemia in the daily life of a person with diabetes will not be reversed more quickly. The practical conclusion that may be drawn from the verbal memory results is that hypoglycaemia impairs the learning of information so that it may be recalled at a later time. For example, if hypoglycaemia occurred while studying, the student would be advised to review material that had recently been examined.

Visual memory was not impaired significantly by hypoglycaemia, but with mean scores of 93 and $82 \%$ for immediate and delayed recall, respectively, during euglycaemia, a ceiling effect was evident. Future work on this aspect of memory should employ tasks with a larger range of items, especially more difficult items.

There was no evidence of a different effect of hypoglycaemia in IHA and NHA subjects. This may have been related to inadequate statistical power for this specific between-subject analysis. However, with a total of 36 subjects and a repeated-measures design, the study was large by comparison with others in this research area. There are some limitations of the between-group comparisons. First, we cannot exclude overlap, even though our method of assessing awareness has been shown to be a good predictor of severe hypoglycaemia at the group level [29]. Subjects 
with similar levels of awareness could receive different classifications as a result of answering with confidence or caution. Second, the two groups were not well matched for other characteristics, which may have confounded the effect of hypoglycaemia on memory performance, particularly the duration of diabetes, the presence of microvascular complications and overall memory performance. For these reasons, the present study cannot exclude a potential difference between people with IHA and NHA.

An important consideration is that studies of this type, which compare group means using a powerful withinsubject design, do not have much power to examine individual differences in the effects of hypoglycaemia on cognition. There is, in our experience, consistent anecdotal evidence from both clinical practice and research to suggest that some individuals are much more susceptible to hypoglycaemia-induced cognitive dysfunction than others. To assess this experimentally is impracticable: for this type of intensive study, an uncommonly large number of subjects would be required. However, given the possibility of inter-individual differences, practical recommendations to people with diabetes should not be too rigid.

In conclusion, the present study has confirmed that there is a disruptive effect of hypoglycaemia on both the specific processes of learning and recall, which is relevant to the behaviour of people with diabetes during daily life. The prospective memory test appears to be a sensitive measure of memory dysfunction during hypoglycaemia that may have greater ecological validity than conventional memory tests, and lends weight to the use of laboratory data to derive practical recommendations for people with diabetes.

Acknowledgements Costs of consumables were supported by Diabetes UK (Edinburgh branch). R. E.Warren and N. N. Zammitt were supported by a research grant from the Juvenile Diabetes Research Foundation. I. J. Deary is the recipient of a Royal Society Wolfson Research Merit Award.

Conflict of interest statement The authors declare that they have no conflict of interest. B. M. Frier has been a member of an advisory panel for, and has received honoraria/consulting fees from, Eli Lilly, Sanofi-Aventis, GlaxoSmithKline and Takeda.

\section{References}

1. Pederson-Bjergaard U, Pramming S, Heller SR et al (2004) Severe hypoglycaemia in 1076 adult patients with type 1 diabetes: influence of risk markers and selection. Diabetes Metab Res Rev 20:479-486

2. Deary IJ (1993) Neuropsychological manifestations. In: Frier BM, Fisher BM (eds) Hypoglycaemia and diabetes: clinical and physiological aspects. Edward Arnold, London pp 337-346

3. Draelos MT, Jacobson AM, Weinger K et al (1995) Cognitive function in patients with insulin-dependent diabetes mellitus during hyperglycemia and hypoglycemia. Am J Med 98:135-144
4. Holmes CS, Koepke KM, Thompson RG, Gyves PW, Weydert JA (1984) Verbal fluency and naming performance in type I diabetes at different blood glucose concentrations. Diabetes Care 7:454-459

5. Mitrakou A, Ryan C, Veneman T et al (1991) Hierarchy of glycemic thresholds for counterregulatory hormone secretion, symptoms, and cerebral dysfunction. Am J Physiol 260:E67-E74

6. Pramming S, Thorsteinsson B, Theilgaard A, Pinner EM, Binder C (1986) Cognitive function during hypoglycaemia in type I diabetes mellitus. Br Med J 292:647-650

7. Widom B, Simonson DC (1990) Glycemic control and neuropsychologic function during hypoglycemia in patients with insulindependent diabetes mellitus. Ann Intern Med 112:904-912

8. Wirsén A, Tallroth G, Lindgren M, Agardh C-D (1992) Neuropsychological performance differs between type 1 diabetic and normal men during insulin-induced hypoglycaemia. Diabet Med 9:156-165

9. Heller SR, Macdonald IA (1996) The measurement of cognitive function during acute hypoglycaemia: experimental limitations and their effect on the study of hypoglycaemia unawareness. Diabet Med 13:607-615

10. Baddeley AD (1996) Human memory: theory and practice, 2nd edn. Psychology, Hove, UK

11. Ewing FME, Deary IJ, McCrimmon RJ, Strachan MW, Frier BM (1998) Effect of acute hypoglycemia on visual information processing in adults with type 1 diabetes mellitus. Physiol Behav 64:653-660

12. McCrimmon RJ, Deary IJ, Huntly BJ, MacLeod KM, Frier BM (1996) Visual information processing during controlled hypoglycaemia in humans. Brain 119:1277-1287

13. Strachan MWJ, Ewing FME, Frier BM, McCrimmon RJ, Deary IJ (2003) Effects of acute hypoglycaemia on auditory information processing in adults with type I diabetes mellitus. Diabetologia 46:97-105

14. McCrimmon RJ, Deary IJ, Frier BM (1997) Auditory information processing during acute insulin-induced hypoglycaemia in nondiabetic human subjects. Neuropsychologia 35:1547-1553

15. Sommerfield AJ, Deary IJ, McAulay V, Frier BM (2003) Moderate hypoglycemia impairs multiple memory functions in healthy adults. Neuropsychology 17:125-132

16. Sommerfield AJ, Deary IJ, McAulay V, Frier BM (2003) Shortterm, delayed and working memory are impaired during hypoglycemia in people with type 1 diabetes. Diabetes Care 26:390-396

17. Deary IJ, Sommerfield AJ, McAulay V, Frier BM (2003) Moderate hypoglycaemia obliterates working memory in humans with and without insulin treated diabetes. J Neurol Neurosurg Psychiatry 74:278-279

18. Sunderland A, Harris JE, Baddeley AD (1983) Do laboratory tests predict everyday memory? A neuropsychological study. J Verbal Learn Verbal Behav 22:341-357

19. Franzen MD, Wilhelm KL (1996) Conceptual foundations of ecological validity in neuropsychological assessment. In: Sbordone RJ, Long CJ (eds) Ecological validity of neuropsychological testing. GR/St Lucie, Delray Beach, FA, pp 91-112

20. Meacham JA, Leiman B (1982) Remembering to perform future actions. In: Neisser U (ed) Memory observed. Freeman, San Francisco, pp 327-336

21. Titov N, Knight RG (2001) A video-based procedure for the assessment of prospective memory. Appl Cogn Psychol 15:61-83

22. Boyle PJ, Nagy RJ, O'Connor AM, Kempers SF, Yeo RA, Qualls C (1994) Adaptation in brain glucose uptake following recurrent hypoglycemia. Proc Natl Acad Sci USA 91:9352-9356

23. Fanelli C, Pampanelli S, Epifano L et al (1994) Long-term recovery from unawareness, deficient counterregulation and lack of cognitive dysfunction during hypoglycaemia, following institution of rational, intensive insulin therapy in IDDM. Diabetologia 37:1265-1276 
24. Fanelli CG, Epifano L, Rambotti A et al (1993) Meticulous prevention of hypoglycemia normalizes the glycemic thresholds and magnitude of most of neuroendocrine responses to, symptoms of, and cognitive function during hypoglycemia in intensively treated patients with short-term IDDM. Diabetes 42:1683-1689

25. Jones TW, Borg WP, Borg MA et al (1997) Resistance to neuroglycopenia: an adaptive response during intensive insulin treatment of diabetes. J Clin Endocrinol Metab 82:1713-1718

26. Mitrakou A, Fanelli C, Veneman T et al (1993) Reversibility of unawareness of hypoglycemia in patients with insulinomas. N Engl J Med 329:834-839

27. Mokan M, Mitrakou A, Veneman T et al (1994) Hypoglycemia unawareness in IDDM. Diabetes Care 17:1397-1403

28. Cryer PE (2004) Diverse causes of hypoglycemia-associated autonomic failure in diabetes. N Engl J Med 350:2272-2279

29. Gold AE, MacLeod KM, Frier BM (1994) Frequency of severe hypoglycemia in patients with type I diabetes with impaired awareness of hypoglycemia. Diabetes Care 17:697-703

30. Lezak MD (1995) Neuropsychological assessment. Oxford University Press, New York

31. Wechsler D (1987) Wechsler memory scale-revised manual. Psychological Corporation, San Antonio

32. Deary IJ, Hepburn DA, MacLeod KM, Frier BM (1993) Partitioning the symptoms of hypoglycaemia using multi-sample confirmatory factor analysis. Diabetologia 36:771-777

33. McAulay V, Ferguson SC, Deary IJ, Frier BM (2001) Acute hypoglycemia in humans causes attentional dysfunction while nonverbal intelligence is preserved. Diabetes Care 24:17451750

34. McAulay V, Deary IJ, Sommerfield AJ, Frier BM (2006) Attentional functioning is impaired during acute hypoglycaemia in people with type 1 diabetes. Diabet Med 23:26-31

35. Evans ML, Pernet A, Lomas J, Jones J, Amiel SA (2000) Delay in onset of awareness of acute hypoglycemia and of restoration of cognitive performance during recovery. Diabetes Care 23:893-897

36. Herold KC, Polonsky KS, Cohen RM, Levy J, Douglas F (1985) Variable deterioration in cortical function during insulin-induced hypoglycemia. Diabetes 34:677-685

37. Blackman JD, Towle VL, Sturis J, Lewis GF, Spire J-P, Polonsky KS (1992) Hypoglycemic thresholds for cognitive dysfunction in IDDM. Diabetes 41:392-399

38. Gold AE, Deary IJ, MacLeod KM, Thomson KJ, Frier BM (1995) Cognitive function during insulin-induced hypoglycemia in humans: short-term cerebral adaptation does not occur. Psychopharmacology 119:325-333

39. Lindgren M, Eckert B, Stenberg G, Agardh C-D (1996) Restitution of neurophysiological functions, performance, and subjective symptoms after moderate insulin-induced hypoglycaemia in non-diabetic men. Diabet Med 13:218-225

40. Fanelli CG, Pampanelli S, Porcellati F et al (2003) Rate of fall of blood glucose and physiological responses of counterregulatory hormones, clinical symptoms and cognitive function to hypoglycaemia in type I diabetes mellitus in the postprandial state. Diabetologia 46:53-64 